\title{
Short Communication Preeclampsia and maternal breast cancer risk by offspring gender: do elevated androgen concentrations play a role?
}

\author{
R Troisi*, ${ }^{*}, 2$, KE Innes ${ }^{3}$, JM Roberts ${ }^{4}$ and RN Hoover' \\ 'Division of Cancer Epidemiology and Genetics, National Cancer Institute, Bethesda, MD, USA; ${ }^{2}$ Department of Community and Family Medicine, \\ Dartmouth Medical School, Hanover, NH, USA; ${ }^{3}$ Center for the Study of Complementary and Alternative Therapies, University of Virginia Health System, \\ Charlottesville, VA, USA; ${ }^{4}$ Magee Womens Hospital, University of Pittsburgh, Pittsburgh, PA, USA
}

Among older mothers, preeclampsia in the first pregnancy was associated with a reduction in maternal breast cancer risk that was significantly more pronounced in women bearing male than female infants. Androgen concentrations in male, preeclamptic pregnancies were consistent with the hypothesis that elevated pregnancy androgens might mediate this apparent modifying effect of fetal gender.

British Journal of Cancer (2007) 97, 688-690. doi: I0.1038/sj.bjc.660392I www.bjcancer.com

Published online 7 August 2007

(c) 2007 Cancer Research UK

Keywords: preeclampsia; androgens; hormones; breast cancer; maternal; offspring gender

The reduced risk of breast cancer observed in mothers subsequent to preeclamptic pregnancies (Polednak and Janerich, 1983; Thompson et al, 1989; Troisi et al, 1998; Vatten et al, 2002; Innes and Byers, 2004; Aagaard-Tillery et al, 2006) is curious and the mechanisms mediating it remain largely unexplored. In their recent paper on preeclampsia/pregnancy-induced hypertension and maternal breast cancer risk, Vatten et al (2007) further characterise this association by offspring's gender, reporting a risk reduction with preeclampsia only when the mother carries a male fetus. The authors conclude that their findings are consistent with a protective effect originating with exposures incurred during the pregnancy rather than to an underlying biological trait of the mother, presumably because such factors would be unrelated to whether she conceives a female or male child. Previous studies (Martin et al, 1986; Steier et al, 2002; Troisi et al, 2003; Atamer et al, 2004) indicate elevations in third trimester circulating maternal androgen concentrations in preeclampsia compared with uncomplicated pregnancies. Maternal exposure to elevated androgen concentrations has been hypothesised to mediate the protective influence of preeclampsia, perhaps by antagonising oestrogen's effects on breast epithelial cells (Innes and Byers, 1999; Cohn et al, 2001).

We attempted to replicate the findings of Vatten et al of greater maternal breast cancer protection associated with male preeclamptic pregnancies using linked birth and tumour registry data from a previous population-based study of early-onset breast cancer (Innes and Byers, 2004). To address the possible role of androgen concentrations in mediating this apparent risk reduction, we re-examined data from a longitudinal study of preeclampsia (Troisi et al, 2003).

* Correspondence: Dr R Troisi, Epidemiology and Biostatistics Program, Division of Cancer Epidemiology and Genetics, National Cancer Institute, 6I20 Executive Blvd, Bethesda, MD 20892-7246, USA;

E-mail: troisin@mail.nih.gov

Received I4 May 2007; revised 21 June 2007; accepted 12 July 2007; published online 7 August 2007

\section{MATERIALS AND METHODS}

\section{New York state-linked birth and tumour registry data}

The study population in this matched case-control study comprised primiparous women with singleton births who completed a first pregnancy in New York state (NY) after 1977 (for detailed description of study population and methods, see Innes and Byers, 2004). To ensure comparability to Vatten et al's study, we included only women with singleton first pregnancies in the present analysis. Cases were 2489 primiparous women who were diagnosed with breast cancer in NY between 1979 and 1995 and at least 1 year after completion of the first pregnancy; age at diagnosis ranged from 22 to 55 years. Controls were 9967 primiparous women matched to cases on county of residence and delivery date and who were not diagnosed with breast or endometrial cancer in NY. Information on factors characterising each woman's first pregnancy was obtained from the birth record of her first-born infant. The association of preeclampsia and maternal breast cancer risk was evaluated using conditional logistic regression (Breslow and Day, 1980).

\section{University of Pittsburgh preeclampsia study}

Data were from a longitudinal preeclampsia study conducted at the University of Pittsburgh (Pittsburgh, PA, USA) that included all women who attended the Magee Womens Hospital's obstetric practice, delivered between February 1994 and May 1998 and agreed to participate. Eligible for analysis were 86 cases and 86 controls from a previous case-control study of preeclampsia with singleton pregnancies and without pre-existing diabetes or hypertension (Troisi et al, 2003). Informed consent for a questionnaire, interview and blood collection was obtained from all study participants. Information on the pregnancy was obtained from medical records. Preeclampsia was defined by explicit criteria (described in Troisi et al, 2003) based on blood pressure 
measurements, proteinuria and hyperuricemia (Chesley, 1985), and all diagnoses were reviewed by a jury of clinical experts.

Maternal blood was collected at admission for labor and delivery. The hormone assays have been described (Troisi et al, 2003). Briefly, unconjugated concentrations of androstenedione and testosterone were measured in serum by radioimmunoassay. The coefficients of variation based on blinded quality control samples were $10.2 \%$ for androstenedione and $9.6 \%$ for testosterone.

The hormones were logarithmically transformed to remove skewness from their distributions. Mean maternal androgen concentrations were determined for preeclampsia and uncomplicated pregnancies by offspring gender using analysis of covariance, and geometric means and 95\% confidence intervals are presented. Per cent difference was derived from the beta estimate of the case/ control (dichotomous) variable in a generalised linear regression model with androgens as the dependent variables. Interactions were tested in linear regression models, with androgens as the dependent variables and case/control status, offspring gender and a product term for case/control status and offspring gender as the independent variables. Statistical significance was defined as $P<0.05$ (two-sided)

\section{RESULTS}

\section{New York state-linked birth and tumour registry data}

Preeclampsia was associated with a modest, non-significant maternal breast cancer risk reduction overall (maternal ageadjusted $\mathrm{OR}=0.87$ ) that was similar to Vatten et al's (2007) overall estimate $(R R=0.86)$, but a marked and significant risk reduction among women who delivered their first infant after the age of 30 years (adjusted $\mathrm{OR}=0.33$ ) (Table 1 ). The overall association of preeclampsia to subsequent breast cancer risk did not differ by

Table I Odds ratios (OR) and 95\% confidence intervals (Cl) for preeclampsia (PE) in the first pregnancy and subsequent maternal breast cancer risk among women 22-55 years of age, New York state, USA

\begin{tabular}{lccl}
\hline & No. of cases/controls with PE & $\mathbf{O R}^{\mathbf{a}}$ & $\mathbf{9 5 \%} \mathbf{~ C l}$ \\
\hline All births & $95 / 386$ & 0.87 & $(0.67-1.13)$ \\
Male infant & $45 / 208$ & 0.86 & $(0.60-1.22)$ \\
Female infant & $50 / 178$ & 0.90 & $(0.64-1.27)$ \\
p interaction 0.86 & & & \\
& & & \\
Maternal age $>30$ & $46 / 79$ & 0.33 & $(0.16-0.65)$ \\
$\quad$ Male infant & $22 / 42$ & 0.18 & $(0.04-0.87)$ \\
Female infant & $24 / 37$ & 0.52 & $(0.13-2.0)$ \\
P interaction 0.01 & & & \\
\hline
\end{tabular}

${ }^{a}$ Odds ratio adjusted for maternal age. offspring gender (adjusted $\mathrm{OR}=0.86$ vs 0.90 for women who delivered a son or a daughter, respectively). However, among the mothers older than age 30 , the risk reduction associated with preeclampsia was significantly greater for those who carried a male infant (adjusted $\mathrm{OR}=0.18$ ) than female infant (adjusted $\mathrm{OR}=0.52) \quad(P$ for interaction $=0.01) \quad($ Table 1$)$, although risk reductions were observed for pregnancies involving either gender.

\section{University of Pittsburgh preeclampsia study}

While mean androstenedione and testosterone concentrations were significantly higher in both male and female preeclamptic compared with uncomplicated pregnancies, absolute concentrations in both preeclamptic and uncomplicated pregnancies were significantly higher in those involving males than females (Table 2). Indeed, the concentrations in the uncomplicated male pregnancies (382 for androstenedione and 166 for testosterone) were similar to those in preeclamptic female pregnancies (381 and 161, respectively).

\section{DISCUSSION}

The overall estimates for preeclampsia and breast cancer risk in the present study were similar whether the pregnancy involved a male or female fetus, consistent with findings from a recent casecontrol study conducted in Long Island, NY (Terry et al, 2007). However, among women who were older at first birth ( $>30$ years), the risk reduction associated with a male fetus was significantly greater than that associated with a female fetus.

Previous mechanistic investigations regarding the association of preeclampsia with breast cancer risk have focused on hormones with an established role in breast carcinogenesis, including oestrogens (Tamimi et al, 2003; Troisi et al, 2003) and androgens (Troisi et al, 2003). If the findings of Vatten et al (2007) and what we observe in the present study among older mothers of male preeclamptic pregnancies are real, characterising the hormonal profile of preeclamptic pregnancies by fetal gender and maternal age may provide additional insight into the mechanisms underlying the apparent protective effect of preeclampsia.

In the present study, the highest androgen concentrations were observed in male, preeclamptic pregnancies. Consistent with these findings, Steier et al (2002) also showed elevated testosterone concentrations in male preeclamptic pregnancies relative to both female preeclamptic pregnancies and uncomplicated pregnancies; in contrast to our observations, the per cent difference between testosterone in the preeclamptic and uncomplicated pregnancies was higher in male- than in female-bearing pregnancies, perhaps reflecting differences in study sampling times. Collectively, these data indicate higher absolute (and in Steier et al's data, higher relative) androgen concentrations in the maternal circulation of

Table 2 Means for and per cent difference (\% diff) with 95\% confidence interval ( $\mathrm{Cl}$ ) in maternal androgen concentrations between preeclampsia (PE) cases and uncomplicated (control) pregnancies adjusted for weeks of gestation, by offspring gender

\section{Offspring gender}

\begin{tabular}{|c|c|c|c|c|c|c|c|c|c|}
\hline Hormone & \multicolumn{4}{|c|}{ Female } & \multicolumn{5}{|c|}{ Male } \\
\hline Testosterone $\left(\mathrm{ng} \mathrm{dl}^{-1}\right)$ & 161 & 139 & 28.5 & $16.0,42.4$ & 180 & 166 & 11.4 & $2.5,21.2$ & 0.03 \\
\hline
\end{tabular}

${ }^{a}$ Per cent difference (\% diff) derives from the beta estimate of the case/control variable in a generalised linear regression model, with logarithm-transformed values for the maternal androgens as the dependent variables. Interactions were tested in a linear regression model, with logarithm-transformed androgens as the dependent variables and case/control status, offspring sex and an interaction term for case/control status $\times$ offspring sex as the independent variables. ${ }^{b} p$ interaction refers to the comparison of per cent difference in each androgen between PE and control pregnancies by offspring gender. 
preeclamptic pregnancies involving a male fetus compared with those involving a female fetus. This suggests that the observed protective effect of preeclampsia on breast cancer risk may in part rely on meeting or exceeding a specific threshold of exposure to endogenous androgens. Because uncomplicated male pregnancies have higher androgen concentrations than uncomplicated female pregnancies, one might expect offspring gender to be associated with maternal breast cancer risk even in normal pregnancy. However, previous studies have not consistently demonstrated such an association (Olsen and Storm, 1998; Troisi et al, 1998; Hsieh et al, 1999; Cnattingius et al, 2005); thus, if protection is associated with absolute androgen concentration, the threshold for this effect must be above the levels characterising uncomplicated male pregnancies. Whether this is biologically plausible is unclear.

Preeclamptic pregnancies that involve male offspring may also differ in other biological factors, such as human chorionic gonadotropin (hCG) (Steier et al, 2002), which has been shown to be higher in male preeclamptic pregnancies than in female preeclamptic pregnancies or in uncomplicated pregnancies involving either offspring gender. Human chorionic gonadotropin has been linked to reduced breast cancer risk in human populations, and has been repeatedly shown to promote mammary gland differentiation and inhibit neoplastic changes in animal models (Innes and Byers, 1999; Janssens et al, 2007). The significantly greater increases in hCG associated with male preeclamptic pregnancies may also in part account for the higher androgen concentrations observed in both our data and that of Steier et al (2002). Other biological mechanisms have also been proposed to explain the observed protective effect of preeclampsia, including immunological factors (Polednak and Janerich, 1983) and alterations in IGF-1 and other compounds linked to breast cancer initiation and promotion (Innes and Byers, 1999, 2004). More recent speculation has focused on the antiangiogenic profile of women who develop pregnancy-induced hypertension (AagaardTillery et al, 2006).

The possibility that preeclampsia affords greater protection for maternal breast cancer in pregnancies involving male fetuses merits replication in other large population-based studies. If confirmed, evaluation by offspring gender of the biomarkers mentioned above as well as others that are known to be altered in preeclamptic pregnancies may further our understanding of the apparent protective influence of preeclampsia on breast carcinogenesis.

\section{REFERENCES}

Aagaard-Tillery KM, Stoddard GJ, Holmgren C, Lacoursiere DY, Fraser A, Mineau GP, Varner MW (2006) Preeclampsia and subsequent risk of cancer in Utah. Am J Obstet Gynecol 195: 691-699

Atamer Y, Erden AC, Demir B, Koçyigit Y, Atamer A (2004) The relationship between plasma levels of leptin and androgen in healthy and preeclamptic pregnant women. Acta Obstet Gynecol Scand 83: $425-430$

Breslow N, Day N (1980) Statistical methods in cancer research. Volume I. The analysis of case-control studies IARC. Sci Publ 32: 5-338

Chesley LC (1985) Diagnosis of preeclampsia. Obstet Gynecol 65: 423-425

Cnattingius S, Torrång A, Ekbom A, Granath F, Petersson G, Lambe M (2005) Pregnancy characteristics and maternal risk of breast cancer. JAMA 294: $2474-2480$

Cohn BA, Cirillo PM, Christianson RE, van den Berg BJ, Siiteri PK (2001) Placental characteristics and reduced risk of maternal breast cancer. J Natl Cancer Inst 93: 1133-1140

Hsieh C-C, Wuu J, Trichopoulos D, Adami H-O, Ekbom A (1999) Gender of offspring and maternal breast cancer risk. Int J Cancer 81: 335 - 338

Innes KE, Byers TE (1999) Preeclampsia and breast cancer risk. Epidemiology 10: $722-773$

Innes KE, Byers TE (2004) First pregnancy characteristics and subsequent breast cancer risk among young women. Int J Cancer 112: 306-311

Janssens JP, Russo J, Russo I, Michiels L, Donders G, Verjans M, Riphagen I, Van den Bossche T, Deleu M, Sieprath P (2007) Human chorionic gonadotropin (hCG) and prevention of breast cancer. Mol Cell Endocrinol 269: $93-98$

Martin JD, Hähnel E, Hähnel R (1986) Plasma androstenedione in normotensive and hypertensive pregnancy. Steroids 48: 315-329

Olsen J, Storm H (1998) Pregnancy experience in women who later developed oestrogen-related cancers (Denmark). Cancer Causes Control 9: $653-657$

Polednak AP (1995) Pre-eclampsia, autoimmune diseases and breast cancer etiology. Medical Hypotheses 44: 414-418

Polednak AP, Janerich JT (1983) Characteristics of first pregnancy in relation to early breast cancer: a case-control study. J Reprod Med 28: $314-318$

Steier JA, Ulstein M, Myking OL (2002) Human chorionic gonadotropin and testosterone in normal and preeclamptic pregnancies in relation to fetal sex. Obstet Gynecol 100: 552-556

Tamimi R, Lagiou P, Vatten LJ, Mucci L, Trichopoulos D, Hellerstein S, Ekbom A, Adami HO, Hsieh CC (2003) Pregnancy hormones, preeclampsia, and implications for breast cancer risk in the offspring. Cancer Epidemiol Biomark Prev 12: 647-650

Terry MB, Perrin M, Salafia CM, Zhang FF, Neugut AI, Teitelbaum SL, Britton J, Gammon MD (2007) Preeclampsia, pregnancy-related hypertension, and breast cancer risk. Am J Epidemiol 165: $1007-1014$

Thompson WD, Jacobson HI, Negrini B, Janerich JT (1989) Hypertension, pregnancy, and risk of breast cancer. J Natl Cancer Inst 81: 1571 - 1574

Troisi R, Potischman N, Roberts JM, Lykins D, Siiteri P, Hoover RN (2003) Maternal serum hormone concentrations in preeclamptic and uncomplicated pregnancies. Int J Epidemiol 32: 455-460

Troisi R, Weiss HA, Hoover RN, Potischman N, Swanson CA, Brogan DR, Coates RJ, Gammon MD, Malone KE, Daling JR, Brinton LA (1998) Pregnancy characteristics and maternal risk of breast cancer. Epidemiology 9: 641-647

Vatten L, Forman MR, Nilsen TI, Barrett JC, Romundstad PR (2007) The negative association between pre-eclampsia and breast cancer risk may depend on the offspring's gender. Br J Cancer 96: 1436-1438

Vatten LJ, Romundstad PR, Trichopoulos D, Skjærven R (2002) Preeclampsia in pregnancy and subsequent risk for breast cancer. $\mathrm{Br} J$ Cancer 87: 971-973 